\section{Addressing the accuracy of direct-to-consumer genetic testing}

To the Editor: In the recently published article, "Falsepositive results released by direct-to-consumer genetic tests highlight the importance of clinical confirmation testing for appropriate patient care, “1 lead author Stephany TandyConnor and other employees of Ambry Genetics Corporation reported that roughly $40 \%$ of positive results from direct-toconsumer (DTC) genetic testing samples were incorrect.

The authors highlight pertinent challenges regarding genotyping rare variants and determining causal relationships between genetic variants and disease, but it is important to point out that these challenges apply to the entire industry and are not unique to DTC tests. Furthermore, the study findings cannot be considered representative of all DTC test results due to the small sample size and ascertainment bias. The false positive rate claimed by Tandy-Connor et al. is based on only 43 variant findings and does not consider true positives that were not referred to Ambry for follow up or variants that Ambry did not have the technology to test.

As the only company offering consumers direct access to genetic health reports authorized by the US Food and Drug Administration (FDA), 23andMe places the utmost importance on the quality of the interpreted results that we report to our customers. We also feel strongly that individuals have a right to their own genetic information. In addition to the interpreted health reports that 23andMe customers can receive, they can choose to access their raw, uninterpreted genotype data encompassing many more genetic markers than are used in health reports. It is important to understand the distinction between 23andMe health reports and the raw genotyping results interpreted by third parties that were the focus of the article.

Proven accuracy through FDA authorization: 23andMe has demonstrated the accuracy of our genotyping assay and the test results provided in our genetic health risk and carrier status reports through multiple, rigorous FDA reviews. In doing so, we have helped to pioneer a regulatory framework for allowing different types of genetic health information to be directly obtained by, and reported to, consumers. Specific requirements for analytical validity are now established for DTC genetic health tests-higher standards than many clinical tests are subjected to, and we are confident that a positive result provided to a customer in one of 23andMe's genetic health reports is highly accurate. Thousands of samples have been used to evaluate the accuracy and reproducibility of our tests to meet regulatory requirements, showing greater than $99 \%$ concordance compared with Sanger sequencing on each of hundreds of different variants tested. Results for variants that meet these strict requirements should not be confused with uninterpreted raw data that are clearly labeled as "not for medical or diagnostic use" within the 23andMe product.

We are not aware of any peer-reviewed study that has demonstrated the analytical performance of 23 andMe health reports to fall outside of FDA-established requirements. In fact, Tandy-Connor et al. report that all results for the three $B R C A 1$ and BRCA2 pathogenic Ashkenazi Jewish founder variants, as well as the deltaF508 CFTR variant for cystic fibrosis, were confirmed by clinical testing. While we always recommend confirmatory clinical testing, we would not want individuals or healthcare professionals to ignore or discount clinically relevant, accurate results from 23andMe's FDAauthorized tests.

Differences in technology do not necessarily affect analytical accuracy: Tandy-Connor et al. also highlight differences in comprehensiveness and methodologies used between DTC and clinical tests as a way to explain purported high false positive rates. However, the technology, in this case, has little bearing on the likelihood of false positives.

Sequencing is not inherently more accurate than microarray genotyping and is impacted by many of the same factors that can affect genotyping assays-for example, structural variation, homology, and depth of coverage. If one were to compare sequencing with targeted genotyping, one might even observe a higher rate of false positives with sequencing, because increasing the search area increases the likelihood of obtaining an incorrect result. Also, sequencing can introduce false positives due to the challenges of interpretation, compared with targeted genotyping assays looking only for well-established pathogenic variants.

Individuals have benefited from greater access to their own health information: On the other side of the discussion posed by Tandy-Connor et al. are the 26 variants (a majority of the variant findings studied) that were confirmed to be accurate. If it were not for DTC testing, the individuals with those variants most likely would not have received this important knowledge about their health. Similarly, studies have found that roughly half of women carrying a pathogenic BRCA1 or BRCA2 variant would not qualify for clinical testing under current guidelines. ${ }^{2}$ Broadening access to testing allows more of these individuals to learn about, and take steps to manage, serious genetic health risks. Indeed, we have customers who shared 23andMe health reports with healthcare professionals who ordered confirmatory testing, and ultimately initiated interventions. In cases involving positive $B R C A$ founder pathogenic variants risk-reducing strategies 
were employed, ${ }^{3}$ and in cases involving genetic conditions such as alpha-1 antitrypsin deficiency, treatments were initiated.

23andMe's mission is to help people access, understand, and benefit from the human genome. As such, we stand behind our decision to provide users with access to all of their data, including their uninterpreted raw data-with appropriate labeling that highlights its intended uses and limitations. We have also long recognized that the availability of unvalidated raw genetic data can affect individuals and healthcare professionals, especially when interpreted by third parties, and we have taken, and continue to take, steps to increase the overall quality of our raw data.

We continue to focus on empowering consumers with understandable genetic health information that meets FDA requirements for analytical and clinical validity. It is our firm belief that consumer empowerment and access to high-quality information, combined with healthcare professional guidance, will usher in a new era of personalization, prevention, and improved health outcomes.

\section{DISCLOSURE}

All authors are full-time employees of 23andMe, but otherwise declare no conflict of interest.
Shirley $W u, P h D^{1}$, Jeffrey Pollard, $M D^{1}$, Arnab Chowdry, $P h D^{1}$, Richard Scheller, $P h D^{1}$ and Robert Gentleman, $P h D^{1}$

${ }^{1}$ 23andMe, Mountain View California, USA. Correspondence: Shirley Wu (swu@23andme.com)

\section{REFERENCES}

1. Tandy-Connor S, Guiltinan J, Krempely K, et al. False-positive results released by direct-to-consumer genetic tests highlight the importance of clinical confirmation testing for appropriate patient care. Genet Med 2018. https://doi.org/10.1038/gim.2018.38 [published online ahead of print March 22 2018].

2. King MC, Levy-Lahad E, Lahad A. Population-based screening for BRCA1 and BRCA2: 2014 Lasker Award. JAMA 2014;312(11):1091-1092.

3. Steinberg, J. Reader: DNA testing could save lives; it did for me. USA Today. April 1, 2018. https://www.usatoday.com/story/opinion/2018/04/ 01/reader-dna-testing-could-save-lives/477024002/. Accessed May 8, 2018.

Advance online publication xx xx 2018. doi:10.1038/s41436-018-0094-5 